A003: Electroclinical Predictors of Cognitive and Seizure Outcome in Children with Epileptic Encephalopathy Due to Electrical Status Epilepticus in Sleep (ESES)

Saraf U.U., ${ }^{1}$ Asranna A., ${ }^{1}$ Menon R.N., ${ }^{1}$ Radhakrishnan A., ${ }^{1}$ Manju P., ${ }^{1}$ Vibina V.P., ${ }^{1}$ Cherian A., ${ }^{1}$ Thomas S.V. ${ }^{1}$

${ }^{1}$ Sree Chitra Tirunal Institute of Medical Sciences, Trivandrum, Kerala, India

Background: Epileptic encephalopathy (EE), associated with ESES, with its associated impact on cognition and language, is an important cause of morbidity in children. The effects of various treatment modalities and the factors affecting treatment response are not fully understood.

Methods: Case records of patients admitted in the institute and diagnosed to have EE with ESES pattern on EEG were accessed. Spike and wave index (SWI) was calculated from sleep records. Language development was assessed using Receptive-Expressive Emergent Language Scale and seizure outcome using the modified Engel seizure score.

Results: Fifty-two children with age ranging from 1 to 19 years were included (idiopathic ESES, $n=19$ and symptomatic ESES with pre-existing developmental delay and/or structural brain lesions, $n=33$ ). The two groups differed in terms of younger age at seizure onset in symptomatic ESES $(p=0.006)$, early age at language regression $(p=0.046)$, history of neonatal seizures $(p=0.038$ ) and slowing of background on EEG ( $p=0.024)$. Language regression was noted in $63.5 \%$ of the cohort. Twenty-five (48\%) patients received steroids alone and showed improvement in seizure $(p \leq 0.001)$ and language outcomes at 1 year $(p=0.021)$, while $21(40.3 \%)$ received steroids + IVIgG and showed improvement in seizure outcome $(p=0.002)$ at 1 year. On 1 - year follow-up, seizure remission was noted in 13 (25\%) patients with improvement in seizure score in 32/39 (82\%) patients and language improvement in $60.8 \%$. Patients with normal background on EEG $(p=0.03)$, generalized spikes ( $p=0.05)$, no Fz negative spikes ( $p=0.01$ ), and SWI $<1.70(p=0.004)$ were found to have favorable cognitive outcomes on follow-up. 31/45 (68.9\%) patients had persistent ESES at 1-year follow-up.

Conclusion: Despite clinical differences, idiopathic and symptomatic ESES have similar response to intervention. Addition of IVIgG to steroids improve seizure outcome, but may not have an impact on cognitive outcome, which is determined by EEG variables.

\section{A004: An Ethnographic and Structured Assessment of Treatment-Seeking Attitudes and Behaviors of People with Epilepsy in the Community \\ Archita Chawla, ${ }^{1}$ Rajinder Bansal, ${ }^{1}$ Suman Sharma, ${ }^{1}$ \\ Namita Bansal, ${ }^{1}$ Gagandeep Singh, ${ }^{1}$ Chirag Gupta, ${ }^{1}$ \\ Karan Chouhan ${ }^{1}$ \\ ${ }^{1}$ Dayanand Medical College and Hospital, Ludhiana, Punjab, India}

Introduction: Many people with epilepsy do not accept to the treatment. The reasons for this are not studied.

Aims: To explore factors associated with failure to access available health care treatments by people with epilepsy in an impoverished community in India.
Methods: We reached out to 143 people who were screened positive for epilepsy in door-to-door survey of 60,000 people; their sociodemographic character, outcome, and current status with 240 people who agreed to accept treatment. This was compiled by a structured as well as ethnographic assessment of attitudes of both groups.

Results: Of 143 patients, 29 had relocated, 25 were contacted by individual for assessment, and nine had died over 12 months. Epilepsy was confirmed in 48 (33.57\%). Nonenrolled subjects were more likely to be ethnic Punjabi ( $p=0.0001)$, unemployed ( $p=0.020)$, had income $<$ Rs. 5,386 $(p=0.006)$. In multivariate analysis, ethnic origin, family income, and other socio- economic status were completely associated with treatment acceptance; verbal autopsy findings on the nine people who died will be presented.

Conclusion: People with epilepsy who do not accept treatment are more likely to be of ethnic origin and from lower income strata. Failure to accept treatment is associated with high mortality.

\section{A005: Expression Profile of Histone Deacetylases in Patients with Hippocampal Sclerosis}

Arpna Srivastava, ${ }^{1}$ Aparna Dixit, ${ }^{1,2}$ Ramesh Dodamanni, ${ }^{1,3}$ Jyotirmoy Banerjee, ${ }^{1,4}$ Manjari Thipathi, ${ }^{1,5}$ P. Sarat Chandra ${ }^{1,3}$ ${ }^{1}$ Center of Excellence for Epilepsy, A joint All India Institute of Medical Sciences-National Brain Research Centre Collaboration, New Delhi, India

${ }^{2}$ Dr. B. R. Ambedkar Centre for Biomedical Research, University of Delhi, Delhi, India

${ }^{3}$ Department of Neurosurgery, All India Institute of Medical Sciences, New Delhi, India

${ }^{4}$ Department of Biophysics, All India Institute of Medical Sciences, New Delhi, India

${ }^{5}$ Department of Neurology, All India Institute of Medical Sciences, New Delhi, India

Introduction: Epigenetic mechanisms like altered histone acetylation may have a crucial role in epileptogenesis. Altered histone $\mathrm{H} 3$ and $\mathrm{H} 4$ acetylation has been demonstrated in experimental models of temporal lobe epilepsy (TLE). Epigenetic histone deacetylation inhibition prevents the development and persistence of TLE in animal models. This study was designed to investigate the changes in the expression of the histone deacetylases (HDACs) in the surgically resected tissue specimens of hippocampal sclerosis (HS) patients with the aim to decipher its role in epileptogenesis.

Methods: For this study, surgically resected tissue specimens of 23 patients were obtained. We have used histopathologically normal hippocampus tissues (17) obtained from the postmortem cases without any history of seizures or other neurological disorders as nonepileptic controls. mRNA levels of HDACs were evaluated by quantitative real-time PCR. HDAC activity and the levels of significantly altered HDACs were measured spectrophotometrically.

Results: A significant increase in mRNA level of HDAC1 (9.02 \pm 2.97 fold, $p=0.029$ ), HDAC4 (4.17 \pm 1.23 fold, $p=0.046)$, HDAC5 ( $7.05 \pm 2.40$ fold, $p=0.036)$, HDAC6 (9.35 \pm 2.35 fold, $p=0.017)$, HDAC10 (9.02 \pm 2.97 fold, $p=0.021)$, and 